\title{
Arrow-Debreu Equilibria With Asymptotically Heterogeneous Expectations Exist ${ }^{\dagger}$
}

\author{
Frank Riedel* \\ Institut für Wirtschaftstheorie I \\ Humboldt-Universität zu Berlin
}

\section{Summary}

In infinite horizon economies only local equivalence of beliefs is needed to ensure the existence of an Arrow-Debreu equilibrium. In fact, agents can even disagree completely in the long run in the sense that asymptotically, their beliefs are singular.

Keywords: Heterogeneous expectations, Arrow-Debreu equilibrium

JEL subject classification: D51, D84

*Spandauer Strasse 1, 10178 Berlin, Germany, Tel. +49 2093 5773, Fax +49 2093 5619, email: riedel@wiwi.hu-berlin.de.

$\dagger$ Support of Deutsche Forschungsgemeinschaft through SFB 373, "Quantification and Simulation of Economic Processes" is gratefully acknowledged. 


\section{Introduction}

In sequential economies under uncertainty with a finite time horizon, equivalence of beliefs is, in general, necessary for the existence of an equilibrium (cf. Harrison and Kreps (1979)). If information is generated by a finite sequence of independent, identically distributed random variables, the marginal distributions must be equivalent under all agents beliefs if an equilibrium exists.

A natural question is whether this property extends to an infinite horizon economy. If the existence of an equilibrium implied that beliefs are equivalent on the $\sigma$-field generated by the infinite sequence of random variables, then one could invoke the Law of Large Numbers to conclude that the marginal distributions were identical. With an infinite time horizon, the existence of an equilibrium would thus require the homogeneity of beliefs — a much stronger requirement than mere equivalence.

However, as it is shown here, this is not the case. In infinite horizon economies where information is generated by a sequence of iid random variables, only equivalence of the marginal distributions (or local equivalence of beliefs) is needed to ensure the existence of an Arrow-Debreu equilibrium. Agents need not agree completely about the probabilities of finite-time events. In fact, they can even disagree completely in the long run in the sense that asymptotically, their beliefs are singular.

To give an example. Consider an economy where uncertainty is generated by an infinite sequence of coin flips. Agent A beliefs that the coin is fair(probability of tail is one half), whereas agent B beliefs that this probability is $\frac{3}{4}$. By the Law of Large Numbers, both agents disagree completely in the long run because agent A believes that the relative frequency of tails 
converges to $\frac{1}{2}$, whereas agent $\mathrm{B}$ thinks that this limit is $\frac{3}{4}$. This does not preclude the existence of an equilibrium, however.

The reason for the result is as follows. Heterogeneous, yet locally equivalent beliefs are a special case of state-dependent (or, as Kreps (1988) suggests to call it, additively-separable-across-states) utility functionals. Conditions for the existence of equilibria in this case are well known. Here, the result of Dana (1993) is applied.

Araujo and Sandroni (1999) claim that "if agents posterior beliefs do not eventually become homogeneous then an equilibrium does not exist." Since this looks like a contradiction, an explanation is in order. Araujo and Sandroni (1999)'s result hinges on a specific assumption made about bankruptcy. The fee $f$ for bankruptcy is exogenously given and independent of equilibrium consumption prices. Therefore, an agent who goes bankrupt if the event $A$ occurs, expects to pay a fee $f P_{i}(A)$ for going bankrupt. This fee can thus be arbitrarily small, as long as the belief of the agent assigns a sufficiently low probability to the event. This property of Araujo and Sandroni (1999)'s model induces agents to go systematically bankrupt if they differ in beliefs. Since bankruptcy cannot occur in equilibrium, agents must have homogeneous beliefs in Araujo and Sandroni (1999). Their result is thus triggered by the specific bankruptcy rule of their model.

\section{Model and Result}

We describe a competitive economy with two agents who hold distinct beliefs about some sequence of identically distributed, independent random variables. 
Let $S=\left\{s_{1}, \ldots, s_{N}\right\}$ be a finite set and

$$
X_{n}: S^{\mathbb{N}} \rightarrow S
$$

the $n$th projection from the space of sequences $S^{\mathbb{N}}$ to $S$. The history up to time $t$ is given by the $\sigma$-field

$$
\mathcal{F}_{t}=\sigma\left(X_{1}, \ldots, X_{t}\right)
$$

$\mathcal{F}_{0}$ is the trivial $\sigma$-field. The overall history is collected in

$$
\mathcal{F}_{\infty}=\sigma\left(X_{1}, X_{2}, \ldots\right)
$$

Two agents choose a consumption plan $c=\left(c_{t}\right)$ out of the choice set

$$
\mathcal{X}:=\left\{c: c \text { bounded, nonnegative, }\left(\mathcal{F}_{t}\right) \text {-adapted process }\right\} \text {. }
$$

The agents hold beliefs

$$
P_{1}=Q_{1}^{\mathbb{N}}, \quad P_{2}=Q_{2}^{\mathbb{N}}
$$

for two probability measures $Q_{i}$ on $S$. Hence, under $P_{i},\left(X_{t}\right)$ is a sequence of i.i.d. random variables with marginal distribution

$$
P_{i}\left[X_{1}=s_{j}\right]=Q_{i}\left(\left\{s_{j}\right\}\right)=: q_{i j}
$$

Assumption 2.1 (Local Equivalence) $\quad$ (i) The probability measures $Q_{1}$ and $Q_{2}$ are equivalent, that is

$$
\forall j=1 \ldots n \quad q_{1 j}>0 \Longleftrightarrow q_{2 j}>0
$$

(ii) $Q_{1} \neq Q_{2}$, that is, $q_{1 j} \neq q_{2 j}$ for some $j=1, \ldots, n$. 
Due to the preceding assumption, the density $\frac{d Q_{1}}{d Q_{2}}$ exists and will be denoted by $q(x)$. In particular, $q\left(s_{j}\right)=\frac{q_{1 j}}{q_{2 j}}$.

As is well known (see, e.g., Harrison and Kreps (1979)), Assumption 2.1 is, in general, necessary for the existence of an equilibrium. Agents agree on which finite-time events are possible and which not. They do not necessarily assign the same probabilities to them, however. Indeed, the Law of Large Numbers implies in this setting, that agents will disagree completely in the long run:

Lemma 2.1 The beliefs $P_{1}$ and $P_{2}$ are locally equivalent. In particular, the density of $P_{1}$ with respect to $P_{2}$ on $\mathcal{F}_{t}$ exists and is given by

$$
d_{t}:=\left.\frac{d P_{1}}{d P_{2}}\right|_{\mathcal{F}_{n}}=\prod_{t=1}^{n} q\left(X_{t}\right) .
$$

The beliefs $P_{1}$ and $P_{2}$ are asymptotically singular, i.e. there is an event $B \in \mathcal{F}_{\infty}$ with

$$
P_{1}(B)=0 \quad \text { and } \quad P_{2}(B)=1
$$

Proof : The $\sigma$-field $\mathcal{F}_{t}$ is generated by the events

$$
A=\left\{X_{1}=x_{1}, \ldots, X_{t}=x_{t}\right\}
$$

for some $x_{k} \in S$. By definition of $P_{i}$,

$$
\begin{aligned}
P_{i}(A) & =\prod_{k=1}^{t} P_{i}\left[X_{k}=x_{k}\right] \\
& =\prod_{k=1}^{t} Q_{i}\left(\left\{x_{k}\right\}\right),
\end{aligned}
$$

and the latter expression is strictly positive for $P_{1}$ if and only if it is strictly positive for $P_{2}$. Thus, $P_{1}$ and $P_{2}$ are equivalent when restricted to $\mathcal{F}_{t}$, or locally equivalent. 
On the other hand, we have $q_{1 j} \neq q_{2 j}$ for some $j$. The Law of Large Numbers implies that

$$
P_{1}\left[\lim _{t \rightarrow \infty} \frac{1}{t} \sum_{k=1}^{t} 1_{\left\{X_{k}=s_{j}\right\}}=q_{1 j}\right]=1
$$

and

$$
P_{2}\left[\lim _{t \rightarrow \infty} \frac{1}{t} \sum_{k=1}^{t} 1_{\left\{X_{k}=s_{j}\right\}}=q_{1 j}\right]=0 .
$$

We assume that both agents have a standard time-additive von NeumannMorgenstern utility functional,

$$
u_{i}(c)=E_{i} \sum_{t=0}^{\infty} \delta_{i}^{t} U_{i}\left(c_{t}\right), \quad i=1,2 .
$$

The expectation is taken with respect to the probability measure $P_{1}$ resp. $P_{2}$.

\section{Assumption $2.2 \quad$ (i) $0<\delta_{i}<1$.}

(ii) The felicity functions $U_{i}:[0, \infty[\rightarrow \mathbb{R}, i=1,2$ are strictly concave and strictly increasing. On ]0, $\infty[$, they are twice continuously differentiable and have infinite marginal felicity at zero: $\lim _{x \downarrow 0} U_{i}^{\prime}(x)=\infty$.

We show next that von Neumann-Morgenstern utilities with heterogeneous, locally equivalent beliefs are a special case of state-dependent preferences. Let $P=\frac{1}{2}\left(P_{1}+P_{2}\right)$. Since $P_{1}$ and $P_{2}$ are locally equivalent, so are $P$ and $P_{i}, i=1,2$. The densities on $\mathcal{F}_{t}$ are

$$
d_{1 t}:=\left.\frac{d P_{1}}{d P}\right|_{\mathcal{F}_{t}}=\frac{2 d_{t}}{1+d_{t}}
$$


and

$$
d_{2 t}:=\left.\frac{d P_{2}}{d P}\right|_{\mathcal{F}_{t}}=\frac{2}{1+d_{t}} .
$$

Note that $0<d_{i t} \leq 2$. Since consumption plans $c \in \mathcal{X}$ are adapted, we can write the utility functionals as

$$
u_{i}(c)=E \sum_{t=0}^{\infty} \delta_{i}^{t} d_{i t} U_{i}\left(c_{t}\right),
$$

where the expectation is taken with respect to $P$. Defining for a state $(s, t) \in$ $S^{\mathbb{N}} \times \mathbb{N}$ the state-dependent utility function $v_{i}$ as

$$
v_{i}((s, t), c):=\delta_{i}^{t} d_{i t}(s) U_{i}(c)
$$

we obtain

$$
u_{i}(c)=E \sum_{t=0}^{\infty} v_{i}\left((s, t), c_{t}(s)\right)
$$

In this sense, the utility functionals of agents have the von Neumann-Morgenstern form under the same probability measure $P$, but with a state-dependent utility function. ${ }^{1}$

Assumption 2.3 Agents are endowed with some $\omega_{i} \in \mathcal{X}$ which are uniformly bounded away from zero.

Definition 2.1 An Arrow-Debreu equilibrium with complete final disagreement is given by a bounded price process $\psi=\left(\psi_{t}\right)$ and an allocation $\left(x_{1}, x_{2}\right) \in$ $\mathcal{X}^{2}$ such that

(i) agents are rational: $x_{i}$ maximizes utility $u_{i}$ over the budget set

$$
\left\{c \in \mathcal{X} ; E \sum_{t=0}^{\infty} \psi_{t}\left(c_{t}-\omega_{i t}\right) \leq 0\right\},
$$

\footnotetext{
${ }^{1}$ This fact is well known. For a textbook reference, cf. (Kreps 1988, Ch.7).
} 
(ii) and markets clear: $x_{1}+x_{2}=\omega_{1}+\omega_{2}$.

Theorem 2.1 An Arrow-Debreu equilibrium with complete final disagreement exists.

Proof : The proof is done by checking the conditions of Theorem 2.5 of Dana (1993) who establishes existence of an Arrow-Debreu equilibrium for state-dependent utilities. To this end, we show that our model is included in Dana's setup.

Let $\mathcal{O}$ be the $\sigma$-field on $S^{\mathbb{N}} \times \mathbb{N}$ which is generated by all $\left(\mathcal{F}_{n}\right)$-adapted processes. Denote by $\zeta$ the counting measure on $\mathcal{N}$. In Dana's notation, we are working with the measure space

$$
(\Omega, \mathbb{F}, \mu):=\left(S^{\mathbb{N}} \times \mathbb{N}, \mathcal{O}, P \otimes \zeta\right)
$$

Note that the utility functions can be written in the form

$$
u_{i}(c)=\int_{\Omega} v_{i}\left((s, t), c_{t}(s)\right) \mu(d s, d t) .
$$

Conditions (i), (iii) and (iv) of Dana (1993) follow directly from our Assumption 2.2. This assumption and the fact that the densities $d_{i t}$ are bounded by 2 imply also

$$
v_{i}((s, t), c) \leq 2 U_{i}(c) \leq 2\left(U_{i}(1)+U_{i}^{\prime}(1)(c-1)\right) .
$$

Hence, also the linear growth condition (ii) of Dana is satisfied.

It remains to check the integrability condition $(E)$ of Dana. The processes $\left(U_{i}^{\prime}\left(\omega_{i t}\right)\right)$ are bounded since endowments are bounded away from zero by our Assumption 2.3. In light of Remark 2.2 of Dana, this means that Condition (E) is satisfied. 
We may therefore apply Theorem 2.5 of Dana to obtain an allocation $x_{i} \in \mathcal{L}^{1}(\Omega, \mathbb{F}, \mu), i=1,2$ and a price process $\psi \in \mathcal{L}_{+}^{\infty}(\Omega, \mathbb{F}, \mu)$ which form an equilibrium. Since Dana works with a larger choice space, it remains to show that $x_{i} \in \mathcal{X}$. This follows from $x_{i} \leq \omega_{1}+\omega_{2}$, which is bounded by Assumption 2.3.

\section{References}

Araujo, A., And A. Sandroni (1999): "On the Convergence to Homogeneous Expectations when Markets Are Complete," Econometrica, 67(3), 663-672.

DAnA, R. (1993): "Existence and Uniqueness of Equilibria when Preferences Are Additively Separable," Econometrica, 61, 953-957.

Harrison, J., And D. KReps (1979): "Martingales and Arbitrage in Multiperiod Security Markets," Journal of Economic Theory, 20, 381-408.

Kreps, D. (1988): Notes on the Theory of Choice, Underground Classics in Economics. Westview Press.

Dept. of Economics, Humboldt University, Spandauer Straße 1, 10178 Berlin, Germany, riedel@wiwi.hu-berlin.de 